\title{
Extragalactic Cosmic Rays
}

\section{Kachelrieß*}

Institutt for fysikk, NTNU, Trondheim, Norway

I review the status of ultrahigh-energy cosmic ray (UHECR) physics. After introducing the main experimental results and summarizing possible intepretations, I discuss observational and theoretical constraints on the sources of UHECRs. I comment also briefly on the role of magnetic fields. Combining these constraints, I argue that luminuous and numerous AGN types as FR-I and Seyfert galaxies, or alternatively hypernovae, are the most promising UHECR sources. Finally, I sketch few of the models presented at the conference before concluding.

37th International Cosmic Ray Conference -ICRC2021-

15-22 July 2021

Online - Berlin, Germany

\footnotetext{
*Presenter
} 


\section{Introduction}

A review with the title "Extragalactic Cosmic Rays" requires first to clarify the lowest energies to be included, what in turn depends on the question where the transition between Galactic and extragalactic CRs takes place. I will later argue that this transition happens around $5 \times 10^{17} \mathrm{eV}$. To be definite, I call ultra-high energy cosmic rays (UHECR) all particles with energies above $10^{17} \mathrm{eV}$. I start with a discussion of the basic experimental results in Sec. 2, before I comment briefly on the role of magnetic fields in Sec. 3. Then observational and theoretical constraints on the sources of UHECRs are discussed in Sec. 4. Finally, I sketch a small selection of the UHECR models presented at this ICRC.

\section{Observations and their interpretation}

Please find this section in the complete version of this contribution available on the arxiv: https: //arxiv.org/abs/2201.04535

\section{Remarks on the role of magnetic fields}

The strength of the Galactic and extragalactic magnetic fields plays a crucial role for the identification of UHECR sources: In addition to the obvious effect of deflections, the resulting time-delays determine the effective source density of transient sources. Moreover, these delays may undermine the selection of potential UHECR sources based on properties of their electromagnetic spectrum, if the delays become larger than the activity periods of the sources.

Galactic magnetic field Tess Jaffe discussed extensively our knowledge about the GMF in her review talk [44]. It is therefore sufficient to note here that the various GMF models differ by putting emphasis on rotation measures of extragalactic sources or of Galactic pulsars. The former models should therefore provide generally a better description of the magnetic field in the Galactic halo, while the latter should perform better in the Galactic plane. However, for none of these GMF models, the coherence length and the (relative) strength of the regular and the turbulent field are constrained such that the escape time of Galactic cosmic rays reproduces the measured secondaryto-primary ratios below the knee. Consequently, these models cannot be used to investigate, e.g., the transition between Galactic and extragalactic cosmic rays or the knee without adjusting these parameters [45].

Extragalactic magnetic field The origin of the EGMF is one of the outstanding questions in astrophysics. Its seed fields may be generated in the primordial universe or by astrophysical processes like galactic plasma outflows. If the field strength of the EGMF is normalised in both cases such to reproduce observations in the cores of galaxy cluster, their filling factors differ drastically. Observationally, the strength of the EGMF is limited independent of its creation mechanism by $2 \times 10^{-9} \mathrm{G}$ from rotation measures [46], while the present strength of fields with a primordial origin is restricted to $5 \times 10^{-11} \mathrm{G}$ from $\mathrm{CMB}$ anisotropies [47]. The existence of hot spots in the UHECR flux, if they can be firmly established, provides an alternative way to derive 
upper limits on the EGMF. There exists also lower limits on the strength and the filling factor of the EGMF [48]. However, it has been argued that they are invalidated by plasma instabilities [49].

If the average deflection angle per correlation length $L_{\mathrm{c}}$ is small, $R_{\mathrm{L}} \gg L_{\mathrm{c}}$, the CR propagation resembles a random walk in the small-angle regime and the variance of the deflection angle after the distance $d$ is given by

$$
\vartheta_{\mathrm{rms}} \equiv\left\langle\vartheta^{2}\right\rangle^{1 / 2} \simeq \frac{\left(2 d L_{\mathrm{c}} / 9\right)^{1 / 2}}{R_{\mathrm{L}}}=25^{\circ} Z\left(\frac{10^{19} \mathrm{eV}}{E}\right)\left(\frac{d}{100 \mathrm{Mpc}}\right)^{1 / 2}\left(\frac{L_{\mathrm{c}}}{1 \mathrm{Mpc}}\right)^{1 / 2}\left(\frac{B}{10^{-9} \mathrm{G}}\right) .
$$

The increased path-length compared to straight-line propagation leads to the time-delay

$$
\Delta t \simeq \frac{d \vartheta_{\mathrm{rms}}^{2}}{4}=1.5 \times 10^{3} \mathrm{yr} Z^{2}\left(\frac{10^{20} \mathrm{eV}}{E}\right)^{2}\left(\frac{d}{10 \mathrm{Mpc}}\right)\left(\frac{L_{\mathrm{c}}}{1 \mathrm{Mpc}}\right)\left(\frac{B}{10^{-9} \mathrm{G}}\right)^{2}
$$

of charged CRs relative to photons [50,51]. This increase can result in the formation of a magnetic horizon [52, 53]: The maximal distance a CR can travel is in the diffusion picture given by $r_{\text {hor }}^{2}=\int_{0}^{t_{0}} \mathrm{~d} t D(E(t))$ where $t_{0}$ is the source age. If we assume that a magnetic field with correlation length $L_{\mathrm{c}} \sim \mathrm{Mpc}$ and strength $B \sim 0.1 \mathrm{nG}$ exists in a significant fraction of the Universe, then the size of the magnetic horizon at $E=10^{18} \mathrm{eV}$ is $r_{\text {hor }} \sim 100 \mathrm{Mpc}$. Hence, similar to the GZK effect at high energies, we see a smaller and smaller fraction of the Universe for lower CR energies. As a consequence, the spectrum of extragalactic CRs visible to us hardens and the extragalactic component becomes suppressed.

\section{Sources, general constraints and their modelling}

\subsection{Observational constraints}

The observed UHECR intensity fixes the required emissivity $\mathcal{L}$ of the UHECR sources, i.e. their energy input per volume and time, up to a model-dependent factor of order one. For the fit presented in Fig. ??, the PAO determined the emissivity above the ankle, $E>5 \times 10^{18} \mathrm{eV}$, at the present epoch as $\mathcal{L} \simeq 6 \times 10^{44} \mathrm{erg} /\left(\mathrm{Mpc}^{3} \mathrm{yr}\right)$. If the transition to extragalactic CRs is early, as we argued above, the corresponding emissivity increases by at least one order of magnitude. For concreteness, we will use $\mathcal{L}=1 \times 10^{46} \mathrm{erg} /\left(\mathrm{Mpc}^{3}\right.$ yr) for the following estimates. In the case of a unique source class, the relation $\mathcal{L}=L n_{s}$ implies that in a plot of luminosity $L$ vs. number density $n_{s}$ potential source classes have to sit along a fixed diagonal. If several source classes contribute significantly to the total emissivity, they can lie below this diagonal.

The absence of small-scale clustering in the UHECR arrival directions implies that the source density_or deflections in magnetic fields-are sufficiently large. Performing an autocorrelation analysis of the PAO events with $E>70 \mathrm{EeV}$, the bounds $n_{s}>5 \times 10^{-4} / \mathrm{Mpc}^{3}$ for a separation angle $\vartheta=3^{\circ}$ and $n_{s}>6 \times 10^{-6} / \mathrm{Mpc}^{3}$ for $\vartheta=30^{\circ}$ were derived $^{1}$ in Ref. [54]. At these energies, sources should be within $200 \mathrm{Mpc}$ and one does not expect from Eq. (1) deflections larger than $20^{\circ}$ even for the strongest allowed field. Thus we will use the limit $n_{s}>3 \times 10^{-5} / \mathrm{Mpc}^{3}$ for the density of UHECR sources. For comparison, the density of the X-ray selected powerful AGNs with X-ray

\footnotetext{
${ }^{1}$ As these limits constitute strong constraints on possible UHECR sources, an update and a more detailed study of the impact of the EGMF are highly desireable.
} 


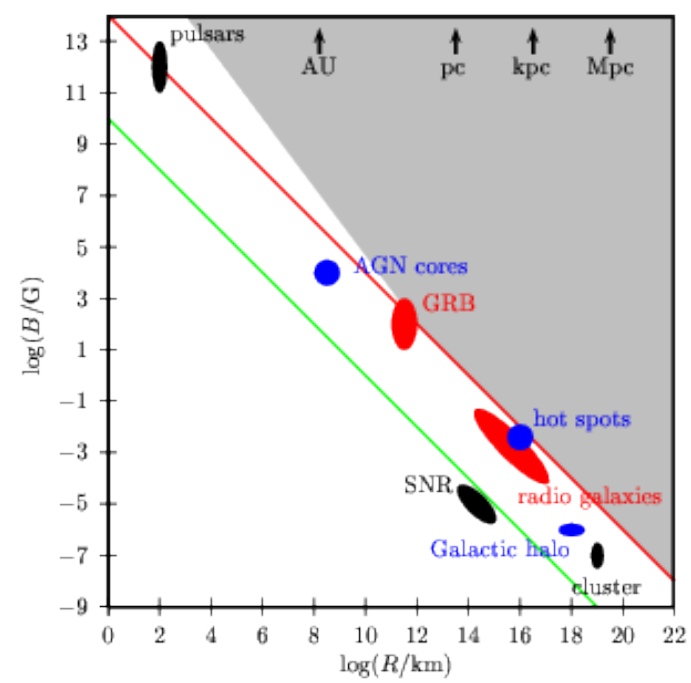

Figure 1: Magnetic field strength $B$ versus size $R$ of various CR sources; adapted from Refs. [56, 57].

luminosity $L>10^{43} \mathrm{erg} / \mathrm{s}$ in the energy range $(0.2-5) \mathrm{keV}$ equals $n_{s} \sim(1-5) \times 10^{-5} / \mathrm{Mpc}^{3}$ within redshift $z \lesssim 0.02$, while the number of Seyfert galaxies is a factor 20 higher [55]. Normal galaxies have a density of $n_{s} \sim 10^{-2} / \mathrm{Mpc}^{3}$.

\subsection{Constraints from theory}

Potential sources of UHECRs have to satisfy as two general constraints the Hillas and Blandford conditions. Combined with measurements of the present energy density of UHECRs and the absence of small-scale anisotropies, the Blandford criterium leads to stringent constraints on the number density of these sources.

Hillas condition The Larmor radius $R_{L}=E /(Z e B)$ of accelerated particles has to fit inside the accelerator of size $R_{S}$ which confines the particles by a magnetic field with strength $B$, i.e. $R_{L}=E /(Z e B) \leq R_{s}$. For known magnetic fields and source sizes, one can constrain thus the maximal achievable energy as $E_{\max }=\Gamma Z e B R_{s}$, a constraint shown in the Hillas plot of Fig. 1 for a compilation of potential cosmic ray sources. The Lorentz factor $\Gamma=\left(1-\beta^{2}\right)^{1 / 2}$ introduced in $E_{\max }$ accounts for a possible relativistic bulk motion of the source and is probably only for gamma-ray bursts and blazars a significant correction. Sources able to accelerate protons to $E>10^{20} \mathrm{eV}$ should lie above the solid red line, while sources above the green line can accelerate iron up to $10^{20} \mathrm{eV}$. Clearly, a heavy composition of UHECRs alleviates considerably the acceleration problem of UHECRs. The main uncertainty in this plot is the strength of the source magnetic field, since nonlinear processes typically lead to an amplification of magnetic fields inside the source. This effect is taken partly into account in Fig. 1, but its exact magnitude is uncertain. Synchrotron energy losses reduce the allowed area for protons to the grey area. In addition, the finite acceleration time disfavours large sources. Thus in general, sources neither too small (minimizing energy losses) nor too big (avoiding too long acceleration times) are favored. 
Blandford condition Constraints on the minimal luminosity of a source able to accelerate CRs up to a certain energy have been derived for specific source types and acceleration mechanisms [58-61], starting from the work of Lovelace [58] on radio galaxies. Blandford stressed first the universality of these limits in Ref. [59], and we will call this bound on the luminosity of a cosmic ray source therefore the Blandford condition.

Let us illustrate this requirement using the simplest example, the acceleration of charged particles by a regular electric field: The acceleration of protons to the energy $E=10^{20} \mathrm{eV}$ requires the potential difference $U=10^{20} \mathrm{~V}$. What is the minimal power $P$ dissipated by such an accelerator? In order to use the basic equation $P=U I=U^{2} / R$ learnt in high-school, we have to know the appropriate value of the resistance $R$. Since an acceleratar operates at densities close to vacuum, we use $R \sim 1000 \Omega$ (guided by the "impedance of the vacuum", $R_{0}=4 \pi k_{0} / c=1 /\left(\epsilon_{0} c\right) \simeq 377 \Omega$ ). Hence a source able to produce protons with $E=10^{20} \mathrm{eV}$ by acceleration in a regular electromagnetic field has the minimal luminosity $L=U^{2} / R \gtrsim 10^{37} \mathrm{~W}=10^{44} \mathrm{erg} / \mathrm{s}$. Including the effect of possible bulk motions and relaxing the maximal energy, the bound becomes

$$
L \gtrsim 3 \times 10^{42} \mathrm{erg} / \mathrm{s} \frac{\Gamma^{2}}{\beta}\left(\frac{E / Z}{5 \times 10^{18} \mathrm{eV}}\right)^{2} .
$$

How does this derivation apply, e.g., to diffusive shock acceleration? This case corresponds to a "circular accelerator", with an energy gain at each crossing of the shock front. Microscopically, the electric field in the fluid frame which is generated by the plasma flow in the shock region accelerates charged particles at each crossing of the shock region. Thus the same argument as above applies. Note also that $R_{0}=c \mu_{0}$ transforms $P=U^{2} / R_{0}$ into an equivalent (assuming the limit $\beta \sim 1$ ), often used form involving the magnetic energy density.

The bound (3) on $L$ can be used to obtain an upper limit on the density $n_{s}$ of UHECR sources, since the observed UHECR intensity fixes the required emissivity $\mathcal{L}=n_{s} L$. Hence, the density of stationary UHECR sources able to accelerate protons to $E=5 \times 10^{18} \mathrm{eV}$ should be smaller than $n_{s}<\mathcal{L} / L \simeq 1 \times 10^{-4} / \mathrm{Mpc}^{3} \Gamma^{-2}$.

\subsection{Combined constraints on the luminosity and number density of UHECR sources}

Stationary sources We combine now in the left panel of Fig. 2 for stationary sources the lower bound on $n_{s}$ to avoid small-scale clustering, $n_{s}>3 \times 10^{-5} / \mathrm{Mpc}^{3}$ (for the case of strong EGMFs), the lower bound on the luminosity and the requirement to reproduce the observed emissivity. The first condition excludes the grey area on the left, while the second requirement excludes (assuming $\Gamma=1$ ) additionally the yellow area at the bottom. Only one source class, FR-I radio galaxies, satisfy both constraints: Taking into account in addition their non-zero bulk Lorentz factors, their luminosity is above the Blandford limit.

How could these conditions be relaxed to allow for more potential UHECR sources? If a second source populations is responsible for the the extragalactic CR flux below the ankle, then the requirement on the emissivity of the first UHECR population is reduced by a factor 20 . In addition, the total energy dissipated in electromagnetic radiation is larger than in the $X$-ray band used as our proxy, $L_{\mathrm{em}}=L_{X}+\ldots$ Adapting these changes promotes also Seyfert galaxies or low-luminosity AGNe to possible UHECR sources. 

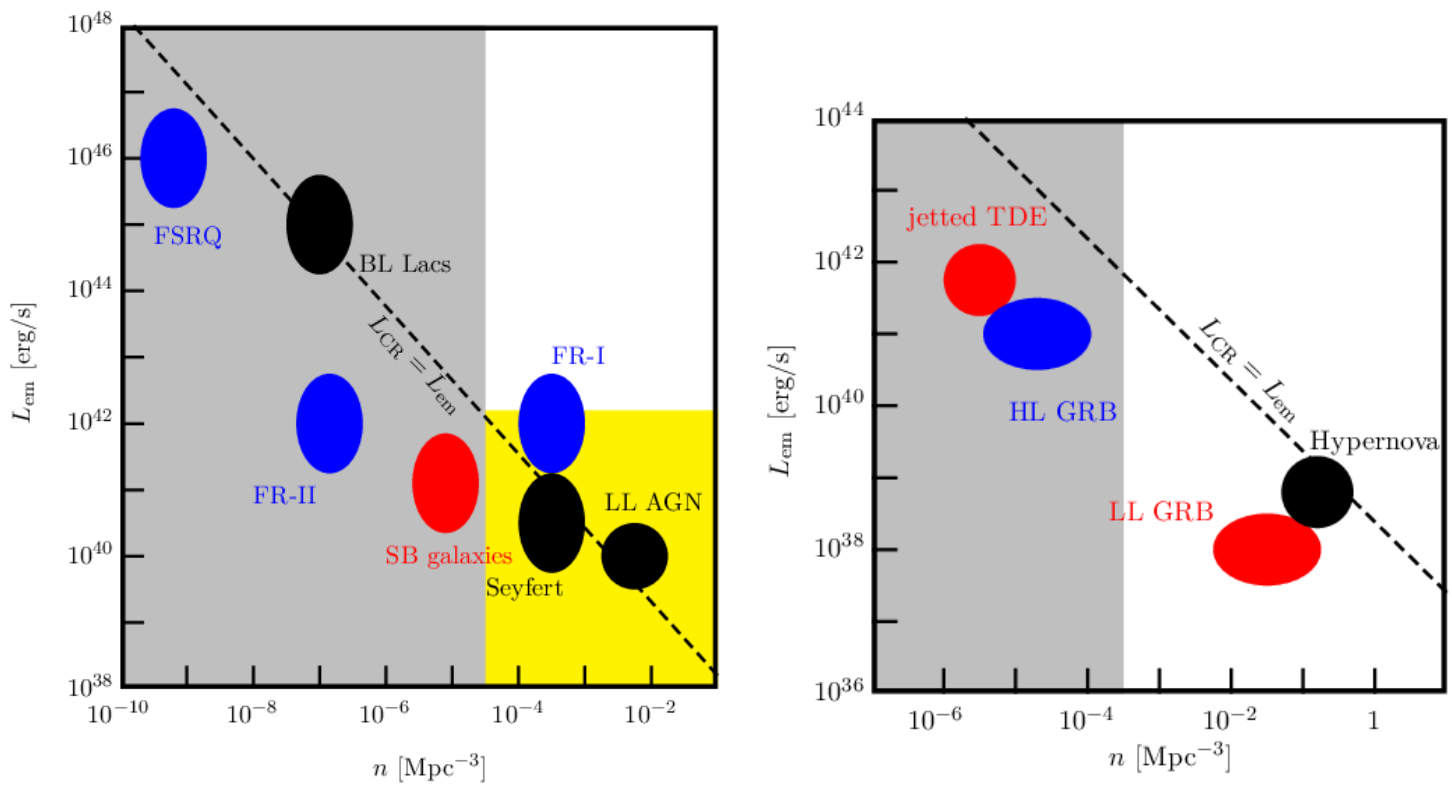

Figure 2: Combined constraints in the luminosity vs. number density plot shown for stationary (left) and transient (right) sources. The light-gray area is excluded by the absence of multiplets, the yellow area by the minimal luminosity argument.

Bursting sources The effective number density of bursting sources with rate $R$ and observed burst duration $\tau$ is given by $n_{s} \simeq 3 R \tau / 5$ [62]. The burst duration $\tau$ is dominated by the time delays in magnetic fields: Using $E=70 \mathrm{EeV}$, i.e. the lower energy used to bound $n_{s}$ from small-scale clustering, together with $B=10^{-11} \mathrm{G}$ gives $\tau \simeq 6 \times 10^{4} \mathrm{yr}$ and $R=5 n_{s} /(3 \tau) \gtrsim 3 \times 10^{-9} / \mathrm{Mpc}^{3} / \mathrm{yr}$. The luminosity bound is for bursting sources as GRBs not a severe restriction, since the true burst time is much smaller than the observed duration $\tau$. However, since these sources are rare, they can typically not provide the observed UHECR emissivity, i.e. they are below the line $L_{\mathrm{CR}}=L_{\mathrm{em}}$ [2]. An exception are hypernovae. In addition, Ref. [63] argued that hypernovae can accelerate protons up to $10^{19} \mathrm{eV}$, if their ejecta have a rather flat energy distribution, $E_{k} \propto(\beta \Gamma)^{-2}$ (compared to $E_{k} \propto(\beta \Gamma)^{-5}$ for non-relativistic shocks).

\subsection{Comments on the modelling of sources: Individual vs. average sources}

Combined fits to the energy spectrum and the composition of UHECRs use often indentical sources with a continuous spatial distribution. In this idealisation, one replaces sources which typically differ even within a specific source class in properties like their CR luminosity, maximal energy, and initial composition by an "average source" plus a redshift evolution of the emissivity. As an example how this idealisation affects the interpretation of such fits, one can consider the effect of a distribution of maximal energies [64]: Assuming for concreteness a power-law distribution for the maximal energies of the individual sources, $d n / d E_{\max } \propto E_{\max }^{-\beta}$, and a power law for the energy spectrum of individual sources, $d N_{i} / d E \propto E^{-\alpha}$, then the total energy spectrum of all sources becomes $d N_{\text {tot }} / d E \propto E^{1-\alpha-\beta}$ in the limit of $E_{\max } \rightarrow \infty$, if they are otherwise identical. Thus the inferred slope agrees with the one of individual sources only for the special case $\beta=1$. 
A distribution of luminosities and maximal energies will reduce the number of active sources at the highest energies. For a smaller number of sources, the likelihood to find small breaks in the spectrum will increase. In this picture, it is natural that new features like the "instep" show up in the energy spectrum as experimental errors are reduced.

\section{Specific models}

\subsection{Active galactic nuclei}

Radio galaxies Fanaroff-Riley radio galaxies are long-standing candidates for UHECR sources. In addition to the well-established types of FR-I and FR-II galaxies, FR-0 galaxies as their lowluminosity extension were suggested in Ref. [65] as accelerator of UHECRs. These authors argued that FR-0 galaxies have the required emissivity to power the observed UHECR energy density, while their rather high density would lead to an almost isotropic conribution to the UHECR flux. Their average jet luminosity of $10^{42}-10^{43} \mathrm{erg} / \mathrm{s}$ is above the Blandford limit. However, acceleration up to the highest observed energies is possible only if hybrid acceleration, i.e. a combination of first-order Fermi with gradual shear acceleration, is realized in these sources.

Blazars Gamma-ray blazars have attracted special attention, since IceCube detected several events from the direction of TXS 0506+056 and PKS 1502+106; see the highlight talk of Foteini Oikonomou for an extensive discussion [66]. An example for these works is Ref. [67], which modelled the populations of low- and high-luminosity blazars together with FSRQs. The measured spectrum and composition of UHECRs is roughly reproduced in this model above $10^{18} \mathrm{eV}$. At the same time, the resulting neutrino fluxes produced inside the AGN jets can obey the IceCube stacking limits in the PeV range, while still giving a significant flux of $\mathrm{EeV}$ neutrinos.

NGC 1068 The Seyfert II galaxy NGC 1068 has attracted also special attention, because of a possible excess of IceCube events [68]. At the same time, MAGIC [69] reported an upper limit to the gamma-ray flux above $200 \mathrm{GeV}$, requiring that the gamma-rays accompanying the neutrino flux must be strongly attenuated in the source. The authors of Ref. [70] gave an updated flux prediction for this AGN using the Stecker-Done-Salamon-Sommers AGN core model and argued that it can accommodate the IceCube excess. The authors of Ref. [71] considered instead the inner regions of the wind launched by the accretion disk. They found that $p p$ interactions with gas may explain the observations, if the gas densities are in the range typical for clouds in the broadline region. NGC 1068 is not only an AGN, but also a starburst galaxy. The authors of Ref. [72] stressed the need to model both the AGN core and the circumnuclear starburst region in order to provide a complete description of the non-thermal phenomena of AGN-starburst composite galaxies.

\subsection{Starburst galaxies}

The evidence for a correlation of UHECR arrival directions with the positions of nearby starburst galaxies poses the question if plausible model for UHECR acceleration in these galaxies can be developped. A natural acceleration site is the termination shock of the strong galactic wind expected in starburst galaxies. The maximal energy achievable for protons,

$$
E_{\max } \simeq 10^{17} \mathrm{eV}\left(t_{\mathrm{acc}} / 10^{9} \mathrm{yr}\right)(B / 0.3 \mu \mathrm{G})\left(v_{\mathrm{sh}} / 1000 \mathrm{~km} / \mathrm{s}\right),
$$


depends on the lifetime of the galactic outflows. Typically, they persist only for times much smaller than the age of galaxies, $t_{\mathrm{acc}} \sim(10-100) \mathrm{Myr}$ [73], and thus $E_{\max }$ is too small. The Blandford condition supports this conclusion. In the model of Ref. [74], acceleration of protons to EeV energies require winds with extreme injection power, $L \sim\left(10^{44}-10^{45}\right) \mathrm{erg} / \mathrm{s}$, beyond what is observed in starburst galaxies. Consequently, most works on starburst galaxies at this ICRC concentrated on the acceleration of $\mathrm{CRs}$ up to energies to tens of $\mathrm{PeV}$, and the associated production of neutrinos, see e.g. Refs. [75, 76].

In an alternative view, a starburst galaxy may not be a UHECR accelerator as an entity but due to its high rate of GRBs, hypernovae, or pulsars. An open question is then if starburst galaxies act as a superposition of single sources, or if the specific environment and collective effects are important. In the former case, we recall that the maximal energy achievable in small sources like pulsars is typically reduced relative to the naive expectation, while the emissivity of GRBs is too low. Only hypernovae may satisfy both constraints, and explain in addition the observed correlations with UHECR arrival directions.

\subsection{Gamma-ray bursts}

Gamma-ray bursts became primary candidates as UHECR and high-energy neutrino sources, after the so-called $\Gamma^{2}$ acceleration mechanisms ${ }^{2}$ was suggested $[60,78]$. However, the large escape probability from relativistic shocks after the first cycle restrict the energy gain per cycle to a factor of order unity, making them much less effective accelerators as initially suggested [79, 80]. Moreover, their emissivity $Q \sim 10^{43} \mathrm{erg} / \mathrm{Mpc}^{3} \mathrm{yr}$ is at least a factor ten too low too explain the observed UHECR flux above the ankle. Finally, the prediction that GRBs are also sources of high-energy neutrinos allowed the search of correlations with IceCube events. From the absence of correlations in the neutrino data, lower bounds on the number density of the sources can be derived, excluding effective denisties below $10^{-6} / \mathrm{Mpc}^{3}$ [81]. These constraints from IceCube apply to high-luminosity GRBs and require either a very low $E_{\max }$ or a small baryon load. Either way, this excludes high-luminosity GRBs as major UHECR sources.

As a remedy, it was suggested that low-luminosity GRBs, i.e. GRBs with $L \sim\left(10^{46}-10^{49}\right) \mathrm{erg} / \mathrm{s}$, may be able to power the UHECR flux. In addition to the general problem of the too small GRB emissivity, it is unclear if high enough energies are achievable in these sources which probably contain shocks in the transrelativistic regime. For the special case of GRB 060218, it was argued that effective acceleration is excluded both in the prompt and afterglow phase [82]. On the other hand, the authors of Ref. [83] argued for GRB 980425 that heavy nuclei can reach energies up to $10^{20} \mathrm{eV}$, if the source is rather extended. This variation between different low-luminosity GRBs may indicate that they do not form a single physical source class.

\section{Conclusions}

The field of UHECRs has seen considerable experimental progress in the last decade. The energy spectrum is measured precisely and has revealed a new feature, the instep. The first clear detection of an anisotropy in the UHECR intensity is a dipole with a $6 \%$ amplitude at

\footnotetext{
${ }^{2} \mathrm{~A}$ modern incarnation is the espresso mechanism discussed in Ref. [77] for AGN jets.
} 
energies $\geq 8 \times 10^{18} \mathrm{eV}$ and a direction which points towards the Galactic anticentre. Thus UHECR experiments resolve for the first time the non-uniformity in the local distribution of UHECR sources. Additionally, the PAO claims evidence for a correlation of the UHECR arrival directions with specific types of UHECR sources, most significant with starburst galaxies. However, the smearing angle used in this study is large, and some confusion between different in source classes (e.g. due to AGN-starburst composites) may occur.

Combining experimental and theoretical constraints on the number density and luminosity of UHECR sources, luminuous and numerous AGN types as FR-I and Seyfert galaxies, or alternatively hypernovae, appear as the most promising UHECR sources. In the latter case, the increased hypernovae rate in starburst galaxies may explain the suggested correlation of their positions with UHECR arrival directions.

The determination of the mass composition of UHECRs has progressed too. The PAO composition data suggest the presence of a Peters cycle above $2 \times 10^{18} \mathrm{eV}$, with well separated elemental groups at different energies. Since this scenario requires in the simplest models very hard injection spectra, it should be scrutinised further. In particular, after its extension and upgrade, TA should be able to confirm or to challenge these results. Moreover, the upgrades of TA and the PAO are important steps towards the future identification of a proton (or light) component on an event-by-event basis which is required to improve correlation analyses with specific source classes.

On the theoretical side, it is desireable to abandon the idea of average sources and to develop and to employ instead models which include physically motivated parameters for individual sources. Exploring in detail the multi-messenger connections will be another important step towards understanding the sources of UHECRs. Hypernova explosions and particle acceleration in their trans-relativistic shocks deserve more thorough studies.

\section{Acknowledgments}

It is a pleasure to thank all my collaborators, and in particular Dima Semikoz, for fruitful discussions and work on topics related to this review. I would like to thank also Björn Eichmann and Michael Unger for comments on this article.

\section{References}

[1] Y. Tsunesada, R. Abbasi, T. Abu-Zayyad, M. Allen, Y. Arai, R. Arimura et al., Joint analysis of the energy spectrum of ultra-high-energy cosmic rays as measured at the Pierre Auger Observatory and the Telescope Array, PoS ICRC2021 (2021) 337.

[2] V. Berezinsky, A. Z. Gazizov and S. I. Grigorieva, On astrophysical solution to ultrahigh-energy cosmic rays, Phys. Rev. D74 (2006) 043005 [hep-ph/0204357].

[3] Pierre Auger collaboration, A. Aab et al., Features of the Energy Spectrum of Cosmic Rays above $2.5 \times 10^{18} \mathrm{eV}$ Using the Pierre Auger Observatory, Phys. Rev. Lett. 125 (2020) 121106 [2008.06488].

[4] Pierre Auger collaboration, A. Aab et al., Measurement of the cosmic-ray energy spectrum above $2.5 \times 10^{18} \mathrm{eV}$ using the Pierre Auger Observatory, Phys. Rev. D 102 (2020) 062005 [2008.06486]. 
[5] Pierre Auger collaboration, A. Castellina, Highlights from the Pierre Auger Observatory, PoS ICRC2019 (2021) 004 [1909. 10791].

[6] Pierre Auger collaboration, A. Aab et al., Inferences on mass composition and tests of hadronic interactions from 0.3 to $100 \mathrm{EeV}$ using the water-Cherenkov detectors of the Pierre Auger Observatory, Phys. Rev. D96 (2017) 122003 [1710 . 07249].

[7] Pierre Auger collaboration, J. Bellido, Depth of maximum of air-shower profiles at the Pierre Auger Observatory: Measurements above $10^{17.2} \mathrm{eV}$ and Composition Implications, PoS ICRC2017 (2018) 506.

[8] Telescope Array collaboration, R. U. Abbasi et al., Depth of Ultra High Energy Cosmic Ray Induced Air Shower Maxima Measured by the Telescope Array Black Rock and Long Ridge FADC Fluorescence Detectors and Surface Array in Hybrid Mode, Astrophys. J. 858 (2018) 76 [1801.09784].

[9] T. Bergmann, R. Engel, D. Heck, N. N. Kalmykov, S. Ostapchenko, T. Pierog et al., One-dimensional Hybrid Approach to Extensive Air Shower Simulation, Astropart. Phys. 26 (2007) 420 [astro-ph/0606564].

[10] R. Alves Batista et al., Open Questions in Cosmic-Ray Research at Ultrahigh Energies, Front. Astron. Space Sci. 6 (2019) 23 [1903.06714].

[11] Pierre Auger, Telescope Array collaboration, A. Yushkov, "Report of the Auger-TA Working Group on the Composition of UHECRs.".

[12] M. Unger, G. R. Farrar and L. A. Anchordoqui, Origin of the ankle in the ultrahigh energy cosmic ray spectrum, and of the extragalactic protons below it, Phys. Rev. D92 (2015) 123001 [1505.02153].

[13] M. Kachelrieß, O. Kalashev, S. Ostapchenko and D. V. Semikoz, Minimal model for extragalactic cosmic rays and neutrinos, Phys. Rev. D96 (2017) 083006 [1704. 06893].

[14] E. Guido, P. Abreu, M. Aglietta, J. M. Albury, I. Allekotte, A. Almela et al., Combined fit of the energy spectrum and mass composition across the ankle with the data measured at the Pierre Auger Observatory, PoS ICRC2021 (2021) 311.

[15] Pierre Auger collaboration, M. Unger, Highlights from the Pierre Auger Observatory, PoS ICRC2017 (2018) 1102 [1710.09478].

[16] M. Kachelrieß, Anisotropies and clustering of extragalactic cosmic rays, Nucl. Phys. B Proc. Suppl. 165 (2007) 272 [astro-ph/0610862].

[17] M. Kachelrieß and P. D. Serpico, The Compton-Getting effect on ultra-high energy cosmic rays of cosmological origin, Phys. Lett. B640 (2006) 225 [astro-ph/0605462].

[18] M. Kachelrieß, P. D. Serpico and M. Teshima, The Galactic magnetic field as spectrograph for ultrahigh energy cosmic rays, Astropart. Phys. 26 (2006) 378 [astro-ph/0510444].

[19] A. Cuoco, R. D’Abrusco, G. Longo, G. Miele and P. D. Serpico, The footprint of large scale cosmic structure on the ultrahigh energy cosmic ray distribution, JCAP 01 (2006) 009 [astro-ph/0510765].

[20] M. Kachelrieß and D. V. Semikoz, Clustering of ultrahigh energy cosmic ray arrival directions on medium scales, Astropart. Phys. 26 (2006) 10 [astro-ph/0512498].

[21] Pierre Auger collaboration, A. Aab et al., Observation of a Large-scale Anisotropy in the Arrival Directions of Cosmic Rays above $8 \times 10^{18} \mathrm{eV}$, Science 357 (2017) 1266 [1709.07321]. 
[22] Pierre Auger collaboration, A. Aab et al., Large-scale cosmic-ray anisotropies above 4 EeV measured by the Pierre Auger Observatory, Astrophys. J. 868 (2018) 4 [1808. 03579].

[23] P. Erdogdu et al., The Dipole anisotropy of the 2 Micron All-Sky Redshift Survey, Mon. Not. Roy. Astron. Soc. 368 (2006) 1515 [astro-ph/0507166].

[24] Telescope Array, Pierre Auger collaboration, P. Tinyakov et al., The UHECR dipole and quadrupole in the latest data from the original Auger and TA surface detectors, PoS ICRC2021 (2021) 375 [2111. 14593].

[25] A. di Matteo, L. A. Anchordoqui, T. Bister, J. Biteau, L. Caccianiga, R. M. de Almeida et al., UHECR arrival directions in the latest data from the original Auger and TA surface detectors and nearby galaxies, PoS ICRC2021 (2021) 308.

[26] Pierre Auger collaboration, A. Aab et al., An Indication of anisotropy in arrival directions of ultra-high-energy cosmic rays through comparison to the flux pattern of extragalactic gamma-ray sources, Astrophys. J. 853 (2018) L29 [1801.06160].

[27] Telescope Array, Pierre Auger collaboration, A. di Matteo et al., UHECR arrival directions in the latest data from the original Auger and TA surface detectors and nearby galaxies, PoS ICRC2021 (2021) 308 [2111.12366].

[28] V. Berezinsky, A. Z. Gazizov and S. I. Grigorieva, Dip in UHECR spectrum as signature of proton interaction with CMB, Phys. Lett. B612 (2005) 147 [astro-ph/0502550].

[29] G. Giacinti, M. Kachelrieß, D. V. Semikoz and G. Sigl, Cosmic Ray Anisotropy as Signature for the Transition from Galactic to Extragalactic Cosmic Rays, JCAP 1207 (2012) 031 [1112 . 5599].

[30] A. W. Strong, J. Wdowczyk and A. W. Wolfendale, The gamma-ray background: a consequence of metagalactic cosmic ray origin?, Journal of Physics A Mathematical General 7 (1974) 120.

[31] V. S. Berezinsky and A. Yu. Smirnov, Cosmic neutrinos of ultra-high energies and detection possibility, Astrophys. Space Sci. 32 (1975) 461.

[32] Fermi-LAT collaboration, M. Ackermann et al., The spectrum of isotropic diffuse gamma-ray emission between $100 \mathrm{MeV}$ and $820 \mathrm{GeV}$, Astrophys. J. 799 (2015) 86 [1410 . 3696].

[33] O. E. Kalashev, D. V. Semikoz and G. Sigl, Ultra-High Energy Cosmic Rays and the GeV-TeV Diffuse Gamma-Ray Flux, Phys. Rev. D79 (2009) 063005 [0704 . 2463].

[34] V. Berezinsky, A. Gazizov, M. Kachelrieß and S. Ostapchenko, Restricting UHECRs and cosmogenic neutrinos with Fermi-LAT, Phys. Lett. B695 (2011) 13 [1003 . 1496].

[35] J. Heinze, D. Boncioli, M. Bustamante and W. Winter, Cosmogenic Neutrinos Challenge the Cosmic Ray Proton Dip Model, Astrophys. J. 825 (2016) 122 [1512 . 05988].

[36] M. Kachelrieß and D. V. Semikoz, Cosmic Ray Models, Prog. Part. Nucl. Phys. 109 (2019) 103710 [1904.08160].

[37] Fermi-LAT collaboration, M. Ackermann et al., Resolving the Extragalactic $\gamma$-Ray Background above $50 \mathrm{GeV}$ with the Fermi Large Area Telescope, Phys. Rev. Lett. 116 (2016) 151105 [1511.00693].

[38] V. S. Berezinsky, S. V. Bulanov, V. A. Dogiel and V. S. Ptuskin, Astrophysics of cosmic rays. Amsterdam, Netherlands: North-Holland, 1990. 
[39] M. Kachelrieß, S. Ostapchenko and R. Tomàs, ELMAG: A Monte Carlo simulation of electromagnetic cascades on the extragalactic background light and in magnetic fields, Comput. Phys. Commun. 183 (2012) 1036 [1106. 5508].

[40] W. D. Apel et al., KASCADE-Grande measurements of energy spectra for elemental groups of cosmic rays, Astropart. Phys. 47 (2013) 54 [1306. 6283].

[41] IceCube collaboration, M. G. Aartsen et al., Observation and Characterization of a Cosmic Muon Neutrino Flux from the Northern Hemisphere using six years of IceCube data, Astrophys. J. 833 (2016) 3 [1607.08006].

[42] ICECube collaboration, M. G. Aartsen et al., The IceCube Neutrino Observatory Contributions to ICRC 2017 Part II: Properties of the Atmospheric and Astrophysical Neutrino Flux, 1710.01191.

[43] I. Tamborra, "Multi-Messenger." Rapporteur talk at the "37th International Cosmic Ray Conference", Berlin, 2021.

[44] T. Jaffe, "Constraining Magnetic Fields at Galactic Scales ." Review talk at the "37th International Cosmic Ray Conference", Berlin.

[45] G. Giacinti, M. Kachelrieß and D. V. Semikoz, Reconciling cosmic ray diffusion with Galactic magnetic field models, JCAP 1807 (2018) 051 [1710.08205].

[46] M. S. Pshirkov, P. G. Tinyakov and F. R. Urban, New limits on extragalactic magnetic fields from rotation measures, Phys. Rev. Lett. 116 (2016) 191302 [1504. 06546].

[47] K. Jedamzik and A. Saveliev, Stringent Limit on Primordial Magnetic Fields from the Cosmic Microwave Background Radiation, Phys. Rev. Lett. 123 (2019) 021301 [1804.06115].

[48] Fermi-LAT collaboration, M. Ackermann et al., The Search for Spatial Extension in High-latitude Sources Detected by the Fermi Large Area Telescope, Astrophys. J. Suppl. 237 (2018) 32 [1804.08035].

[49] A. E. Broderick, P. Tiede, P. Chang, A. Lamberts, C. Pfrommer, E. Puchwein et al., Missing Gamma-ray Halos and the Need for New Physics in the Gamma-ray Sky, Astrophys. J. 868 (2018) 87 [1808 . 02959].

[50] E. Waxman and J. Miralda-Escude, Images of bursting sources of high-energy cosmic rays. 1. Effects of magnetic fields, Astrophys. J. 472 (1996) L89 [astro-ph/9607059].

[51] J. Miralda-Escude and E. Waxman, Signatures of the origin of high-energy cosmic rays in cosmological gamma-ray bursts, Astrophys. J. 462 (1996) L59 [astro-ph/9601012].

[52] E. Parizot, GZK horizon and magnetic fields, Nucl. Phys. Proc. Suppl. 136 (2004) 169 [astro-ph/0409191].

[53] V. Berezinsky and A. Z. Gazizov, Diffusion of cosmic rays in expanding universe, Astrophys. J. 643 (2006) 8 [astro-ph/0512090].

[54] Pierre Auger collaboration, P. Abreu et al., Bounds on the density of sources of ultra-high energy cosmic rays from the Pierre Auger Observatory, JCAP 05 (2013) 009 [1305 . 1576].

[55] A. T. Steffen, A. J. Barger, L. L. Cowie, R. F. Mushotzky and Y. Yang, The changing AGN population, Astrophys. J. Lett. 596 (2003) L23 [astro-ph/0308238].

[56] M. Kachelrieß, Lecture notes on high energy cosmic rays, 0801.4376.

[57] K. V. Ptitsyna and S. V. Troitsky, Physical conditions in potential sources of ultra-high-energy cosmic rays. I. Updated Hillas plot and radiation-loss constraints, Phys. Usp. 53 (2010) 691 [0808.0367]. 
[58] R. V. E. Lovelace, Dynamo model of double radio sources, Nature 262 (1976) 649.

[59] R. D. Blandford, Acceleration of ultrahigh-energy cosmic rays, Phys. Scripta T85 (2000) 191 [astro-ph/9906026].

[60] E. Waxman, Cosmological gamma-ray bursts and the highest energy cosmic rays, Phys. Rev. Lett. 75 (1995) 386 [astro-ph/9505082].

[61] M. Lemoine and G. Pelletier, On electromagnetic instabilities at ultra-relativistic shock waves, Mon. Not. Roy. Astron. Soc. 402 (2010) 321 [0904.2657].

[62] K. Murase and H. Takami, Implications of Ultra-High-Energy Cosmic Rays for Transient Sources in the Auger Era, Astrophys. J. Lett. 690 (2009) L14 [0810 . 1813].

[63] X.-Y. Wang, S. Razzaque, P. Meszaros and Z.-G. Dai, High-energy Cosmic Rays and Neutrinos from Semi-relativistic Hypernovae, Phys. Rev. D 76 (2007) 083009 [0705 . 0027].

[64] M. Kachelrieß and D. V. Semikoz, Reconciling the ultra-high energy cosmic ray spectrum with Fermi shock acceleration, Phys. Lett. B 634 (2006) 143 [astro-ph/0510188].

[65] L. Merten, M. Boughelilba, A. Reimer, P. Da Vela, S. Vorobiov, F. Tavecchio et al., FR- 0 jetted active galaxies: extending the zoo of candidate sites for UHECR acceleration, PoS ICRC2021 (2021) 986 [2107. 13278].

[66] F. Oikonomou, High-energy neutrino emission from blazars, PoS ICRC2021 (2021) 030.

[67] X. Rodrigues et al., "Active galactic nuclei as neutrino sources in the PeV and EeV regimes." Contribution 1321 at the "37th International Cosmic Ray Conference", Berlin, 2021.

[68] ICECube collaboration, M. G. Aartsen et al., Time-Integrated Neutrino Source Searches with 10 Years of Ice Cube Data, Phys. Rev. Lett. 124 (2020) 051103 [1910 . 08488].

[69] MAGIC collaboration, V. A. Acciari et al., Constraints on gamma-ray and neutrino emission from NGC 1068 with the MAGIC telescopes, Astrophys. J. 883 (2019) 135 [1906. 10954].

[70] L. A. Anchordoqui, J. Krizmanic and F. Stecker, High-Energy Neutrinos from NGC 1068, POS ICRC2021 (2021) 993.

[71] S. Inoue, M. Cerruti, K. Murase and R.-Y. Liu, High-energy neutrinos and gamma rays from the AGN-driven wind in NGC 1068, PoS ICRC2021 (2021) 1013.

[72] B. Eichmann, R.-J. Dettmar and J. Becker-Tjus, An AGN-starburst composite multi-messenger model of NGC 1068, PoS ICRC2021 (2021) 1006.

[73] G. E. Romero, A. L. Müller and M. Roth, Particle acceleration in the superwinds of starburst galaxies, Astron. Astrophys. 616 (2018) A57 [1801.06483].

[74] E. Peretti, G. Morlino, P. Blasi, P. Cristofari and M. Ahlers, Exploring galactic wind superbubbles by multimessenger observations, PoS ICRC2021 (2021) 995.

[75] A. Condorelli, S. Petrera, D. Boncioli and E. Peretti, Starburst Galaxies as possible sources of UHECRs and neutrinos, PoS ICRC2021 (2021) 959.

[76] A. Marinelli, A. Ambrosone, M. Chianese, D. Fiorillo, G. Miele and O. Pisanti, A novel multimessenger study of Starburst galaxies: implications for neutrino astronomy, PoS ICRC2021 (2021) 1232.

[77] R. Mbarek, D. Caprioli and K. Murase, Ultra-High-Energy Cosmic Rays and Neutrinos from relativistic jets of Active Galactic Nuclei, PoS ICRC2021 (2021) 481.

[78] M. Vietri, On the acceleration of ultrahigh-energy cosmic rays in gamma-ray bursts, Astrophys. J. 453 (1995) 883 [astro-ph/9506081]. 
[79] A. Achterberg, Y. A. Gallant, J. G. Kirk and A. W. Guthmann, Particle acceleration by ultrarelativistic shocks: Theory and simulations, Mon. Not. Roy. Astron. Soc. 328 (2001) 393 [astro-ph/0107530].

[80] M. Lemoine, G. Pelletier and B. Revenu, On the efficiency of Fermi acceleration at relativistic shocks, Astrophys. J. Lett. 645 (2006) L129 [astro-ph/0606005].

[81] M. Kowalski, Status of High-Energy Neutrino Astronomy, J. Phys. Conf. Ser. 632 (2015) 012039 [1411.4385].

[82] F. Samuelsson, D. Begue, F. Ryde, A. Pe'er and K. Murase, The problematic connection between low-luminosity gamma-ray bursts and ultra-high-energy cosmic rays, PoS ICRC2021 (2021) 467.

[83] A. Rudolph, J. Heinze, D. Biehl, A. Fedynitch, D. Boncioli, Z. Bošnjak et al., UHECR from high-and low-luminosity Gamma-Ray Bursts, PoS ICRC2021 (2021) 1000. 